\title{
Trigonometric Functions Above and Below of the Double-Slit Barrier
}

\author{
Jiří Stávek ${ }^{1}$ \\ ${ }^{1}$ Bazovského 1228, 16300 Prague, Czech republic \\ Correspondence: Jiří Stávek, Bazovského 1228, 16300 Prague, Czech republic. E-mail: stavek.jiri@seznam.cz
}

Received: July 15, 2017

Accepted: July 20, 2017

Online Published: July 26, 2017

doi:10.5539/apr.v9n4p59

URL: https://doi.org/10.5539/apr.v9n4p59

\begin{abstract}
The Double-Slit Experiment with individual electrons represents the central mystery of quantum mechanics and was voted by readers of Physics World as the most beautiful experiment in physics. In our trigonometric model we have divided the Young-Feynman double-slit interferometer into two zones: Aristotelian mixing chamber (the space between the entrance slit $S_{0}$ and above the double-slit barrier with slits $S_{1}$ and $S_{2}$ where particles have been reflected (Euclid) and/or randomly deviated - parenklisis of Epicurus) and Plato's projection chamber (the space below the double-slit barrier and above the projection screen). Aristotelian mixing chamber represents the classical hidden variable: individual particles have been reflected and/or randomly deviated in this zone and later pass through the double-slit barrier in such a way that they create probability distributions of single particles described by the very well known trigonometric relations. The double-slit barrier serves as a "self-organizing filter" for reflected and/or deviated individual particles and creates an order in the population densities among individual particles. For the experimental evaluation of this trigonometric model we propose to insert an internal and external "sfumato mirrors" for the quantitative manipulation of probability distributions of individual particles. The concept "sfumato" was inspired by the Old Master Leonardo da Vinci (see his picture Mona Lisa) ("sfumato" means "smoke"). Sfumato mirrors represent the classical observable variable and their influence on the resulting probability densities of individual particles can be trigonometrically described. Sfumato mirrors must not absorb any particles. In another experimental modification we can insert below one slit of the double-slit barrier an "absorption chiaroscuro mask" to fully remove those penetrating particles from the system and thus manipulate with the probability distribution observed with one opened slit ("chiaroscuro" means "light-dark"). It could be of interest to observe the resulting probability distribution of individual particles registered on the projection screen from one opened slit $\mathrm{S}_{1}$ or $\mathrm{S}_{2}$. The absorption chiaroscuro mask must not reflect particles back to the Aristotelian mixing chamber or to the Plato's projection chamber. The results of these experiments will decide if we are just going up in a blind alley".
\end{abstract}

Keywords: Aristotelian mixing chamber, Plato's projection chamber, Leonardo's sfumato mirrors, Rembrandt's chiaroscuro mask, probability distributions of individual particles, Goethe's "More trigonometry!"

\section{Introduction}

The Double-Slit Experiment with individual electrons represents the central mystery of quantum mechanics and recently was voted by readers of Physics World as the most beautiful experiment in physics - see R.P. Crease in 2002.

Richard Feynman in 1965 proposed his version of the Young's double-slit experiment for individual particles and warned his readers: "We should say right away that you should not try to set up this experiment. This experiment has never been done in just this way. The trouble is that apparatus would have to be made on an impossibly small scale to show the effects we are interested in." Richard Feynman video lecture to this topic might be on interest. (R.F. had been illuminated by the Plato's lighting and that is why we can see two his shadows on the chalkboard. Some observers might deduced from these shadows that R.F. was splitted).

During the last 50 years experimentalists made great steps in the realization of this Young-Feynman double-slit experiment: e.g. C. Jönsson in 1961, P.G. Merli et al. in 1976, A. Tonomura et al. in 1989. P.G. Merli et al. and A. Tonomura et al. carried out experiments with a biprism and clearly demonstrated the interference pattern formation of individual electrons. But this technique with a biprism cannot be considered as the true Young-Feynman doubleslit interference experiment. 
The true Young-Feynman double-slit experiment was realized only recently by two groups: G. Matteuci et al. in 2013 and R. Bach et al. in 2013. Both groups realized the double-slit experiment under the condition when electrons travel through the slits in an electromagnetic field free region. A brief history of the development of these techniques can be found in Matteuci in 2013. Roger Bach (2014) made a significant progress in the experimental technique with his movable mask below the double-slit barrier and stated: "One major question is what happens between the source, the double-slit, and the detector."

The recent development of this available experimental technique for the Young-Feynman double-slit experiment enables to check the validity of the second famous quote of Richard Feynman: "We choose to examine a phenomenon which is impossible, absolutely impossible, to explain in any classical way, and which is in the heart of quantum mechanics."

In our proposed trigonometric concept we will employ the knowledge of Old Masters in order to guide us on the tour in this microscale. Aristotelian school could be used for the description of reflected (Euclid) and/or randomly deviated (Epicurus - parenklisis) particles in the so-called Aristotelian mixing chamber (the space between the entrance slit $\mathrm{S}_{0}$ and above the double-slit barrier with slits $\mathrm{S}_{1}$ and $\mathrm{S}_{2}$ ). The allegory of Plato's cave will be used as a model for the Plato's projection chamber (the space below the double-slit barrier and above the projection screen). On our projection screen we observe patterns of particles without a precise knowledge of their arrival paths. The know-how of Plato and Aristotle represents the classical hidden variable in our experiment. Arthur Herman (2014) summarized know-how of those two great philosophers.

In order to manipulate with the arrival paths of electrons and their distribution patterns on the projection screen we propose to insert an internal and external "sfumato mirrors" for the quantitative manipulation of probability distributions of individual particles. The concept "sfumato" ("smoke") was inspired by the Old Master Leonardo da Vinci (see Leonardo's picture Mona Lisa where Leonardo developed his sfumato technique). Sfumato mirrors represent the classical observable variable and their influence on the resulting probability densities of individual particles can be trigonometrically described.

We expect that sfumato mirrors might bring new quantitative information about the travel history of individual particles and might explain why the closing of one slit and insertion of any obstacle into the paths of travelling particles dramatically modify the resulting pattern on the projection screen. The double-slit barrier serves as a "self-organizing filter" for reflected and/or randomly deviated individual particles and creates an order in the population densities among individual particles and together with sfumato mirrors we might manipulate with probability distributions of single electrons.

We have another experimental possibility - to insert below one of the slits $\mathrm{S}_{1}$ or $\mathrm{S}_{2}$ an "absorption chiaroscuro mask" that should absorb all particles penetrating through that slit. This concept "chiaroscuro" ("light-dark") was inspired by pictures of Old Masters as Botticeli, Caravaggio, Vermeer, Rembrandt, Rubens and many others. (See e.g. Rembrandt's picture An Old Man in Red). In this case we filter out selectively all particles from one slit while the particles from the other slit should create one half of the intereference pattern. Details of these old techniques can be found in the classical book of Giorgio Vasari: "There are Plato and Aristotle, and around them is a great school of philosophers."

The modern application of "chiaroscuro" in the black and white photography was developed by George Eastman (e.g. C.W. Ackerman in 1930). In order to achieve the chiaroscuro effect in the photography the "Rembrandt lighting" setup can be designed in studio portrait photography. The photographic lighting significantly influences the impression from photographs, e.g. Hunter, Biver and Fuqua in 2015.

The trigonometric inspiration for this contribution came from Masters who constructed the folding rules and proportional compasses (called as "sectors") between $16^{\text {th }}$ and $18^{\text {th }}$ centuries for the calculation of values of trigonometric functions. E.g., the folding rule made by Antonio Bianchini in 1564 was very inspirative. The Italian sector constructed by Galileo, the French sector constructed by Nicolas Bion, and the English sector constructed by Edmund Stone were very helpful to the students of their time. Introduction to this topic can be found in Monica Blanco in 2016 and in the review of Tomash and Williams.

It could be possible that these newly applied trigonometric functions might guide us back to the old classical physics. We can paraphrase the known quote of Johann Wolfgang Goethe "More light!" as "More trigonometry!"

\section{Oscillating Particles with Wavelength $\lambda$ Travelling through the Double-Slit Barrier}

In this proposed scenario the double-slit barrier acts as a "self-organizing filter" for penetrating particles. When both slits are opened, then the interference structure in the probability densities of single particles appears on the projection screen. When one of those slits $S_{1}$ or $S_{2}$ is closed, then all particles are forced to travel through one slit 
and the selforganization in the probability density disappears. At this moment this "self-organizing filter", represented by the double-slit barrier, is an unknown classical hidden variable.

Based on this unknown classical hidden variable we can further develop the experimentally measured probability densities $\mathrm{P}_{1}(\mathrm{x})$ for the closed slit $\mathrm{S}_{2}$, the probability density $\mathrm{P}_{2}(\mathrm{x})$ for the closed slit $\mathrm{S}_{1}$, and the probability density $\mathrm{P}_{12}(\mathrm{x})$ for both opened slits:

$$
P_{12}(x)=P_{1}(x)+P_{2}(x)+2 \sqrt{P_{1}(x) P_{2}(x)} \cos (\delta)
$$

where $\delta=(2 \pi \mathrm{d} \sin \theta) / \lambda$ is the phase difference between the waves coming from two slits and arriving to the point $\mathrm{x}$ on the projection screen, $d$ is the distance between two slits and $\theta$ is the angle to the straight direction. For details on this probability distribution see the web site of James D. Cresser (2009).

This trigonometric situation is shown in Figure 1. At this moment we are still in the realm of the wave theory and Max Born rule (1926). For the further development of this concept we have to bring a new classical observable parameter beyond this known description.

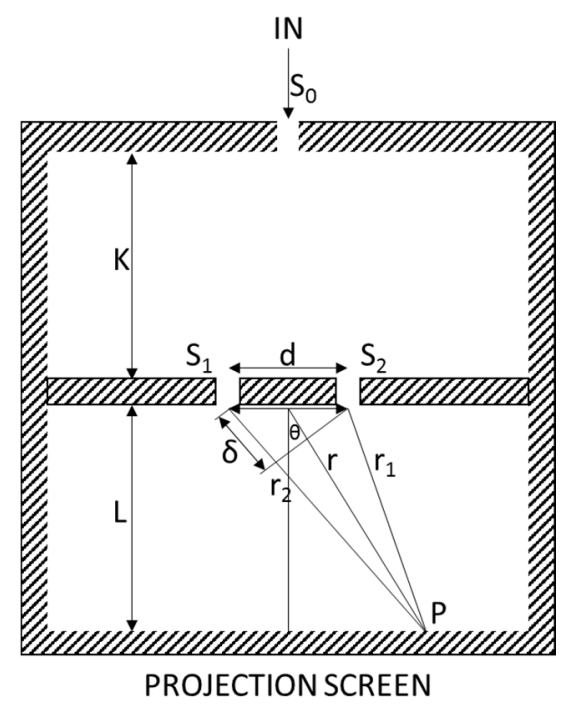

Figure 1. Young-Feynman double-slit experiment with particles oscillating with wavelength $\lambda$

\section{Two Chambers in the Young-Feynman Double-Slit Experiment}

In the Young-Feynman double-slit experiment we can define two zones: the zone between the entrance slit $\mathrm{S}_{0}$ and the double-slit barrier with slits $\mathrm{S}_{1}$ and $\mathrm{S}_{2}$ - we will describe this zone as the Aristotelian mixing chamber. Aristotelian mixing chamber represents the classical hidden variable: individual particles have been reflected and/or randomly deviated in this zone and later pass through the double-slit barrier in such a way that they create probability distributions of single particles described by the very well known trigonometric relations.

The zone between the double-slit barrier and the projection screen we will describe as the Plato's projection chamber - a kind of allegory of the Plato's cave where observers try to guess the reality from shadows. Observers analyzing the probability distribution patters on the projection screen try to evaluate mechanisms of those processes.

It is a long tradition since the time of Thomas Young to depict the travelling waves from the entrance slit $\mathrm{S}_{0}$ through both slits $S_{1}$ and $S_{2}$ to the projection screen only in one direction. In our scenario we will assume that particles might be reflected and/or randomly deviated in Aristotelian mixing chamber and in the combination with the double-slit barrier a kind of selforganization among single particles might occur.

The schema of the Young-Feynman double-slit interferometer is shown in Figure 2. 


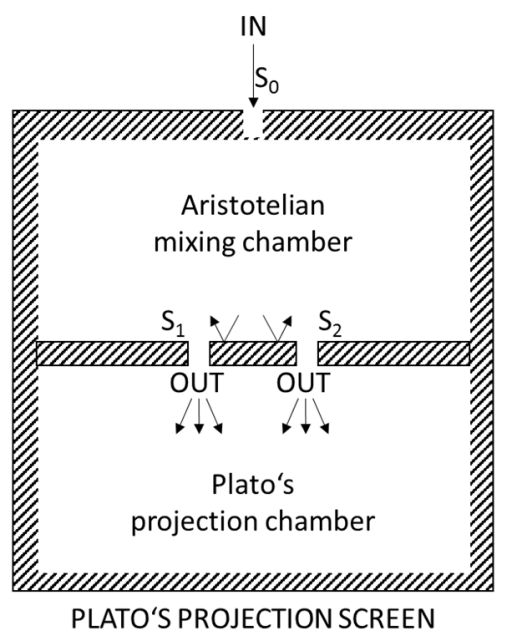

Figure 2. Young-Feynman double-slit experiment with two zones: Aristotelian mixing chamber and Plato's projection chamber. The path of particles between the entrance slit $\mathrm{S}_{0}$ and the projection screen is not known

\section{Leonardo's Sfumato Mirrors}

For the manipulation with particle paths in the Young-Feynman double-slit experiment we propose to insert socalled Leonardo's sfumato mirrors - these mirrors must not absorb those particles. The internal sfumato mirror should be built above the double-slit barrier in the middle of slits while the external sfumato mirror should be built below the double-slit barrier in the middle of slits: Figure 3.

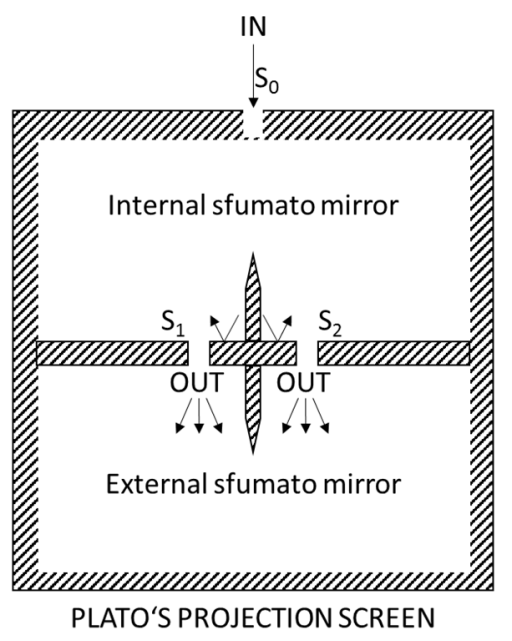

Figure 3. Young-Feynman double-slit experiment modified by classical observable variables: internal sfumato mirror and external sfumato mirror

Figure 4 depicts trigonometric functions that will be used for the quantitative prediction of resulting population densities of single particles on the projection screen. The height of these sfumato mirrors should influence the paths of reflected and/or randomly deviated particles. Therefore, the observed interference structure of single particles on the projection screen should be modified by this classical observable variable.

The symbols in Figure 4: $\mathrm{K}$ is the distance between the slit $\mathrm{S}_{0}$ and the double-slit barrier, $\mathrm{H}_{\alpha}$ is the height of the internal sfumato mirror, $\mathrm{L}$ is the distance between the double-slit barrier and the projection screen, $\mathrm{H}_{\beta}$ is the height of the external sfumato mirror. 


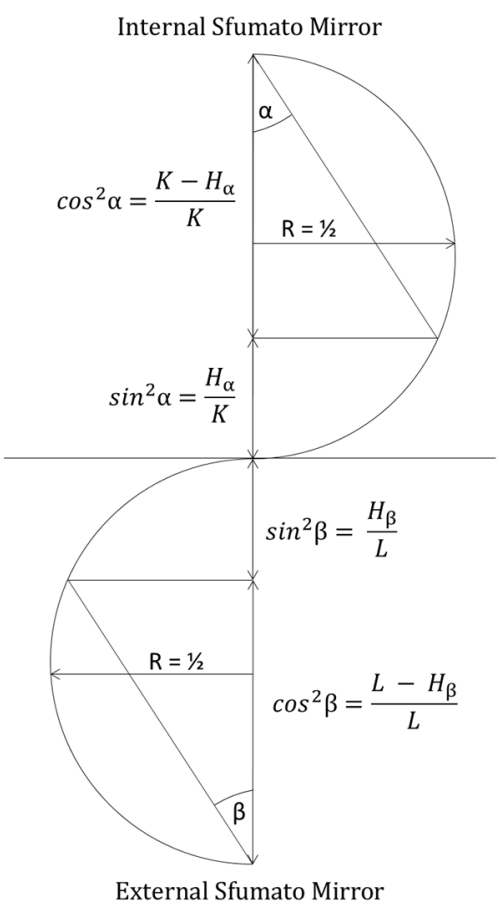

Figure 4. Trigonometric functions describing the influence of the height of internal and external sfumato mirrors on the resulting probability distribution

We predict that the internal sfumato mirror changes the resulting probability density of single particles on the projection screen as:

$$
P_{122 N T}(x)=P_{1}(x)+P_{2}(x)+2 \sqrt{P_{1}(x) P_{2}(x)} \cos (\delta) \cos ^{2}(\alpha)
$$

where $\cos ^{2}(\alpha)$ describes the free space for the free motion of particles in the Aristotelian mixing chamber.

We predict that the external sfumato mirror changes the resulting probability density of single particles on the projection screen as:

$$
P_{12 E T T}(x)=P_{1}(x)+P_{2}(x)+2 \sqrt{P_{1}(x) P_{2}(x)} \cos (\delta) \cos ^{2}(\beta)
$$

where $\cos ^{2}(\beta)$ describes the free space for the free motion of particles in the Plato's projection chamber.

\section{Rembrandt's Chiaroscuro Mask}

Old Masters studied in details the effects of lighting on the resulting interplay of light and shadows in order to achieve a dramatic illumination in their pictures: they developed several techniques as sfumato, chiaroscuro, cangiante, unione, and tenebrism. We can be inspired by those techniques also for the case of the Young-Feynman double-slit experiment and thus create some new structures between light and shadows in the microworld.

We can employ the Rembrandt's chiaroscuro mask below one of slits $S_{1}$ or $S_{2}$ and filter out from the system particles coming through one of those slits. This chiaroscuro mask has to absorb all particles below of those slits - no reflection is allowed back to the Aristotelian mixing chamber or into the Plato's projection chamber. This situation is shown in Figure 5. 


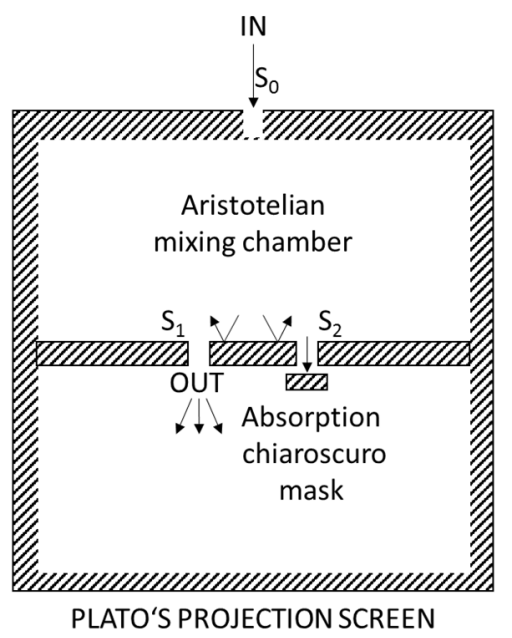

Figure 5. Young-Feynman double-slit experiment modified by a classical observable variable - absorption chiaroscuro mask

Particles transferring through the opened slit $\mathrm{S}_{1}$, while the slit $\mathrm{S}_{2}$ is blocked by the chiaroscuro mask, should form a half of the interference patters on the projection screen:

$$
P_{1,2 \text { CHIA }}(x)=P_{1}(x)+\sqrt{P_{1}(x) P_{2}(x)} \cos (\delta)
$$

Particles transferring through the opened slit $\mathrm{S}_{2}$, while the slit $\mathrm{S}_{1}$ is blocked by the chiaroscuro mask, should form a half of the interference patters on the projection screen:

$$
P_{1 \text { СHH }, 2}(x)=P_{2}(x)+\sqrt{P_{1}(x) P_{2}(x)} \cos (\delta)
$$

This quantitative prediction of observed population densities of single particles on the projection screen might support this concept. It will be a challenge for the experimenters but their skills were recently significantly developed and improved. They might play with light and shadows on this small scale and to bring know-how of Old Maters to the Microworld.

Roger Bach (2014) is now experimentally very close to this concept with his movable mask below the double-slit barrier. Roger Bach used a movable mask covered by a $2 \mathrm{~nm}$ thick layer of gold. We estimate that this mask could be described as the Leonardo's sfumato mirror that reflects electrons back to the Aristotelian mixing chamber or to the Plato's projection chamber. It would be of high interest if the movable mask could be made as the Rembrandt's chiaroscuro mask that removes completely all electrons passing through one of both slits from the system. In this case we might get two halves of the interference pattern coming separately from the slits $\mathrm{S}_{1}$ and $\mathrm{S}_{2}$. A similar prediction was published by G. Cavalleri et al. (2010) based on the other mechanism - the stochastic electrodynamics with spin (SEDS).

The last warning of Richard Feynman: "Do not keep saying to yourself, if you can possibly avoid it,'but how can it be like that?' Because you will get down the drain into a blind alley from which nobody has yet escaped. Nobody knows how it can be like that."

\section{Conclusions}

1) The Young-Feynman double-slit interferometer was modeled with two zones: Aristotelian mixing chamber (classical hidden variable) and Plato's projection chamber.

2) Two classical observable variables were proposed for the experimental analysis of this concept: Leonardo's sfumato mirrors and Rembrandt's chiaroscuro mask.

3) Trigonometric functions for the description of probability densities of single particles for those two new observable variables were proposed.

4) This concept can be tested in Laboratories of actual Leaders in this field. 


\section{Acknowledgments}

This work was supported by the JP\&FŠ Agency (Contract Number 25g/1963), by the VZ\&MŠ Agency (Contract Number 16000/1989) and by the GMS Agency (Contract Number 69110/1992). The valuable inspiration to the Figure 2 was given by ES (Contract Number 314/1992). We have found the valuable support on the web site www.wolframalpha.com with the corrections of used formulae.

\section{References}

Ackerman, C. W. (1930). George Eastman: Founder of Kodak and the Photography Business. Beard Books.

Alberghi, G. L. (2013). Single Electron Interference and Diffraction Experiments with a High Energy Physics Detector. Retrieved from: https://agenda.infn.it/getFile.py/access?contribId=24\&resId=0\&materialId=slides $\&$ confId $=6120$

Bach, R. (2013). Controlled double-slit electron diffraction. New Journal of Physics, 15, 033018.

Bach, R. (2014). Electron Matter Interferometry and the Electron Double-Slit Experiment. PhD Thesis, University Nebraska-Lincoln, Department of Physics and Astronomy. Retrieved from: http://digitalcommons.unl.edu/ physicsdiss $/ 32 /$

Bianchini, A. (1564). Folding Rule. Instituto e Museo di Storia della Scienzia, Firenze, Inventory no.2511.

Blanco, M. (2016). Of the Use of the „English Sector" in Trigonometry: What amount of mathematical training was necessary in the 18th century? History and Pedagogy of Mathematics, July 2016, Montpellier, France. HAL Id: hal-01349245.

Born, M. (1926). Zur Quantenmechanik der Stoßvorgänge. Zeitschrift für Physik, 37, 863-867. https://doi.org/ 10.1007/BF01397477.

Cavalleri, G. (2010). A Quantitative Assessment of Stochastic Electrodynamics with Spin (SEDS): Physical principles and Novel Applications. Front. Phys. China, 5, 107-122.

Crease, R. P. (2002). The Most Beautiful Experiment. Phys. World, 15, 19-20.

Cresser, J. D. (2009). The Two Slit Experiment. Chapter 4, Quantum Physics. Retrieved from: http://physics.mq. edu.au/ jcresser/Phys301/Chapters/Chapter4.pdf

Cresser, J. D. (2009). The Two Slit Experiment. Quantum Physics. Retrieved from: http://docplayer.net/34594182Phys301-quantum-physics-j-d-cresser-rm-c5c357-ext-semester-semester-phys301-quantum-physics-1185.html

Da Vinci, L. (n.d.). Study on Light and Shadow. In Leonardo da Vinci (1452 - 1519). Zöllner, F. \& J. Nathan, Taschen/Slovart. pp. $672-679$.

Feynman, R. (1988). QED: The Strange Theory of Light and Matter. Princeton University Press.

Feynman, R. (2015). The Double-Slit Experiment with Single Electrons. Video presentation. Retrieved from https://www.youtube.com/watch?v=2mIk3wBJDgE

Feynman, R., Leighton, R. B., \& Sands, M. L. (1965). The Feynman Lectures on Physics: Quantum Mechanics. (Vol. 3, chapter 1). Reading, MA: Addison-Wesley.

Frémontier-Murphy, C. (2013). A New Mathematical Vision for an Innovative Calculating Instrument: Galileo's Sector. Bulletin of the Scientific Instrument Society, 118, 35-43.

Galilei, G. (1606). The Operations of the Geometric and Military Compass. Retrieved from https://www.mtholyoke.edu/courses/mpeterso/galileo/compass.html

Herman, A. (2014). The Cave and the Light: Plato Versus Aristotle, and the Struggle for the Soul of Western Civilization. Random House Trade paperbacks.

Hunter, F., Biver, S., \& Fuqua, P. (2015). Light Science and Magic: An Introduction to Photographic Lighting. Focal Press.

Jönsson, C. (1961). Elektroneninterferenzen an mehreren künstlich hergestellten Feinspalten. Z. Phys., 161, 454474.

Matteuci, G. (2013). Build-up of interference patterns with single electrons. Eur. J. Phys., 34, 511-517.

Matteuci, G. (2013). Interference with electrons - from thought to real experiment. Proc. of SPIE Vol. 8785, $8785 \mathrm{CF}-1$. 
Merli, P. G., Missiroli, G. F., \& Pozzi, G. (1976). On the Statistical Aspect of Electron Interference Phenomena. Am. J. Phys., 44, 306-307.

Tomash, E., \& Williams, M. R. (n.d.). The Sector: its History, Scales, and Uses. Retrieved from: http://citeseerx.ist.psu.edu/viewdoc/download?doi=10.1.1.87.8516\&rep=rep1\&type=pdf

Tonomura, A., Endo, J., Matsuda, T., Kawasaki, T., \& Ezawa, H. (1989). Demonstration of Single-electron Buildup of an Interference Pattern. Am. J. Phys., 57, 117-120.

Vasari. G. (2008). The Lives of the Artists. Oxford World's Classics, Oxford University Press.

Young, T. (1807). A Course of Lectures on Natural Philosophy and the Mechanical Arts (Vol. 1, William Savage. Lecture 39, pp. 463-464). https://doi.org/10.5962/bhl.title.22458

\section{Copyrights}

Copyright for this article is retained by the author(s), with first publication rights granted to the journal.

This is an open-access article distributed under the terms and conditions of the Creative Commons Attribution license (http://creativecommons.org/licenses/by/4.0/). 